\title{
Narrative Entertainment System with Tabletop Interface
}

Takashi Mori, Katsutoki Hamana, Chencheng Feng, and Jun'ichi Hoshino

\author{
1-1-\#3M309, Tennodai 1-chome, Tsukuba-shi, Ibaraki, Japan \\ e0611423@edu.esys.tsukuba.ac.jp, \\ \{hamana, feng_chencheng\} @entcomp.esys.tsukuba.ac.jp, \\ jhoshino@esys.tsukuba.ac.jp
}

\begin{abstract}
We propose the Narrative Entertainment System with Tabletop Interface. This system is using a miniature-shaped interface called Physical Character. By recognizing the operation of Physical Character, virtual actor's behavior is under control, and that offers a method to create a story while playing with miniatures in a familiar way since our childhood. In the form of intuitive operation with this interface, you are not only an observer, but also a creator.
\end{abstract}

Keywords: narrative entertainment, tabletop, interface, tangible, interactive storytelling, edutainment.

\section{Introduction}

Storytelling is typical of the narrative entertainment, and is helpful to children in improving their logic, language skill, expressions and communication skills [1].

Storytelling with some interactions gives a chance to enable users to participate in the creation of the story, that has expanded the interests of story experience and reinforced the newness of the story contents. StoryMat[2] uses the toys, which can record children's voice and action for saving and replaying stories when they are playing with. On the other hand, there are researches of interactive systems by operating objects on the table like [3] and [4].

In this paper, we propose a method of Narrative Entertainment System with Tabletop Interface with a miniature-shaped interface in the real world to control the stories and environments of virtual world. In this system, miniatures and dolls with a full length of several centimeters take important part in interface to enable an interaction between users and the story world. Dolls or miniatures are familiar since our childhood, so it is an advantage to make it more intuitive than regular input devices.

As a system to control the process of the story, we have developed a story engine that can manage a huge number of episode segments, and it can actively generate animations of massive character.

\section{System Concept}

The picture in the leftside of Fig. 1 is showing the concept of this system. The story world consists of the part of virtual world and the part of real world. This system has 
an engine to manage the story (Story Engine), several miniatures to control the items or virtual actors (Physical Character), a table-shaped device with a display to get the input data (Tabletop Interface; TTI), and a vertical theater view (Story Monitor). This system is supposed to be placed in a living room. Whenever users watch the animation of life-like characters, they can move the Physical Characters to create their stories actively. By using substantial tangible interfaces such as dolls or miniatures, familiar and intuitive operation becomes possible, and this system is available to encourage children to study. Moreover, it supports a multiple play, which is related to improve children's communication skills.
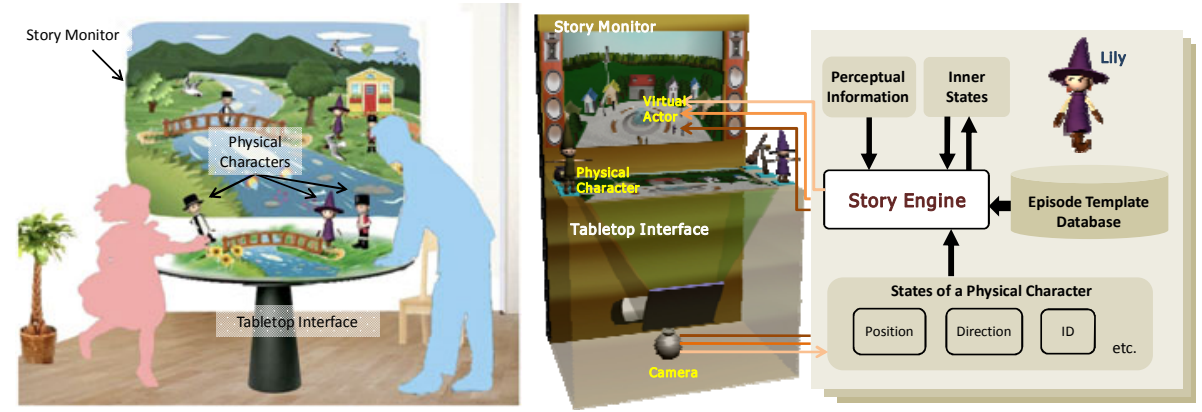

Fig. 1. System Concept Image and System Configuration

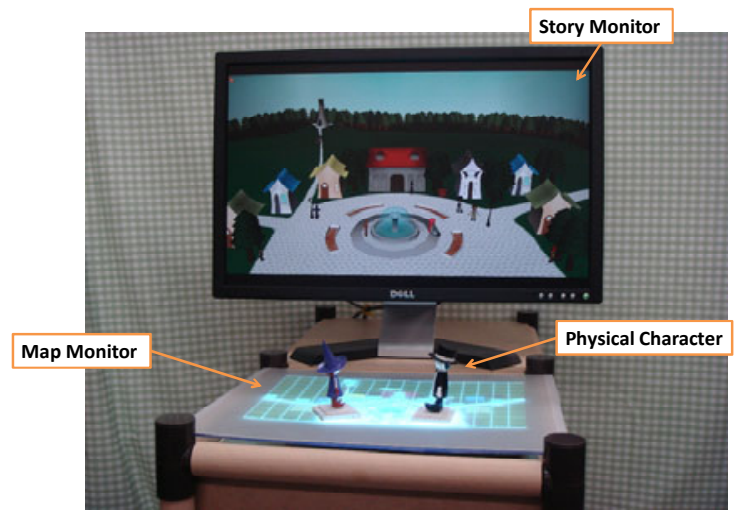

Fig. 2. TTI System Prototype

\section{System Configuration}

The picture in the rightside of Fig. 1 is showing composition of this system. It is mainly consist of 3 parts, an engine to manage the story, a table-shaped device with a display to get the input data, and a vertical display. Each of them are named Story Engine, Tabletop Interface (TTI), and Story Monitor. Moreover, models of virtual characters and virtual items have been used as input device. That is called Physical Character. 
Story Engine controls the Character Status and Story Progression on the basis of the input data of TTI, it shows the status of characters and objects on the Story Monitor. The core system, which controls the whole action of characters to be shown in the story, includes our privious research Massive Action Control System (MACS) [5].

TTI enables a way of creating stories by manipulating Physical Characters. TTI includes Physical Characters, camera, projector and mirror. Position of Physical Characters is recognized with camera and data are sent to Story Engine and reactions are generated to respond user's operation. Fig. 2 shows the system prototype of TTI.

Story Monitor provides a real time view of the virtual world which the users have created. It mainly shows the 3D models of the virtual world, of which corresponded with Physical Character, and other characters living in the story world. Story gets the user's operation and continues the story, which is projected to the Story Monitor to help it easier to understand.

\section{Conclusion}

We proposed a Narrative Entertainment System with Tabletop Interface which can be intuitively operated by children and adults in this paper. By using a miniature-shaped interface, stories can be created while playing with miniatures in a familiar way since our childhood. We have constructed the prototype and tested the system with episode templates and verified the effectiveness. As the result, users evaluated the intuitive way of control and easiness of operation about this interface. However they pointed out the lack of creation of stories.

There are two directions for future development. The first is to develop episode templates. Cooperation and teachings depend on contents, so we are thinking of bring in long stories, or using episodes with clear logic to upgrade and test the episode templates. The second one is to develop the interface. The current TTI is operating in a 2D panel. We are thinking of moving and changing directions miniatures like in the entertainment of Dollhouse, which can support a 3D movement and transformation. It may be updated with 3D sensing and movable Physical Characters.

\section{References}

1. Wright, A.: Creating stories with children. Oxford University Press, Oxford (1997)

2. Ryokai, K., Cassel, J.: StoryMat: A play space with narrative memories. In: Proc. of CHI 1999, Pittsburgh, PA, pp. 272-273 (1999)

3. Kakehi, Y., Yamaoka, J., Akatsuka, D., Naemura, K.: Tablescape Animation: A Basic Study on Supporting Making Animations Using Tabletop Objects. In: Interaction 2009 (2009)

4. Kakehi, Y., Iida, M., Naemura, T., Shirai, Y., Matsushita, M., Ohguro, T.: Lumisight Table: An Interactive View-Dependent Display-Table for Mutiple Users. IEEE Computer Graphics \& Applications 25(1), 48-53 (2005)

5. Hoshino, J., Hamana, K., Shiratori, K., Nakano, A.: Distributed Episode Control System for Interactive Narrative Entertainment. In: Natkin, S., Dupire, J. (eds.) ICEC 2009. LNCS, vol. 5709, pp. 145-156. Springer, Heidelberg (2009) 